A NNALES

UNIVERSITATIS MARIAE CURIE-SKŁODOWSKA

LUBLIN - POLONIA

\title{
The Fekete-Szegö problem for a class of analytic functions defined by Carlson-Shaffer operator
}

\begin{abstract}
In the present investigation we solve Fekete-Szegö problem for the generalized linear differential operator. In particular, our theorems contain corresponding results for various subclasses of strongly starlike and strongly convex functions.
\end{abstract}

1. Introduction. Let $\mathcal{A}$ be the family of all analytic functions $f$ of the form

$$
f(z)=z+\sum_{n=2}^{\infty} a_{n} z^{n}
$$

in the open unit disk $\mathcal{U}=\{z \in \mathbb{C}:|z|<1\}$. Suppose $S$ is a subfamily of $\mathcal{A}$ consisting of functions that are univalent in $\mathcal{U}$. For functions $f, g \in \mathcal{A}$, given by $f(z)=z+\sum_{n=2}^{\infty} a_{n} z^{n}$ and $g(z)=z+\sum_{n=2}^{\infty} b_{n} z^{n}$, we define the Hadamard product (or convolution) of $f(z)$ and $g(z)$ by

$$
(f * g)(z)=z+\sum_{n=2}^{\infty} a_{n} b_{n} z^{n}=(g * f)(z), z \in \mathcal{U} .
$$

Carlson and Shaffer in [4] introduced a linear operator $L(a, c): \mathcal{A} \rightarrow \mathcal{A}$ defined by $L(a, c) f(z)=\phi(a, c ; z) * f(z)$, where the symbol $*$ denotes the

2000 Mathematics Subject Classification. 30C45.

Key words and phrases. Fekete-Szegö problem, Hadamard product, linear operator, strongly starlike functions, strongly convex functions. 
convolution of two functions in $\mathcal{A}$ and where $\phi(a, c ; z)$ is the well-known incomplete beta function given by

$$
\phi(a, c ; z)=z+\sum_{n=2}^{\infty} \frac{(a)_{n-1}}{(c)_{n-1}} z^{n}, z \in \mathcal{U} .
$$

Here $a$ and $c$ are nonzero complex parameters and $a, c \neq-1,-2,-3, \ldots$. Also, $(\lambda)_{n}$ denotes the Pochhammer symbol defined by

$$
(\lambda)_{n}=\frac{\Gamma(\lambda+n)}{\Gamma(\lambda)}= \begin{cases}1, & n=0, \\ \lambda(\lambda+1) \ldots(\lambda+n-1), & n \in\{1,2,3, \ldots\} .\end{cases}
$$

We also note that $L(a, a) f(z)=f(z), L(2,1) f(z)=z f^{\prime}(z)$ and $L(\delta+$ 1,1) $f(z)=D^{\delta} f(z)$, where

$$
D^{\delta} f(z)=\frac{z}{(1-z)^{\delta+1}} * f(z), \delta>-1,
$$

is the generalized Ruscheweyh derivative of function $f$ in $\mathcal{A}$ [22]. The operator $L(a, c)$ is analytic in $\mathcal{U}$ and plays an important role in Geometric Functions Theory; see for example [24], [14], [21] and [9].

The linear multiplier differential operator $D^{m}(\lambda, \varphi) f$ was defined by the authors in [7] as follows:

$$
\begin{aligned}
D^{0}(\lambda, \varphi) f(z) & =f(z), \\
D^{1}(\lambda, \varphi) f(z) & =D(\lambda, \varphi) f(z) \\
& =\lambda \varphi z^{2}(f(z))^{\prime \prime}+(\lambda-\varphi) z(f(z))^{\prime}+(1-\lambda+\varphi) f(z), \\
D^{2}(\lambda, \varphi) f(z) & =D(\lambda, \varphi)\left(D^{1}(\lambda, \varphi) f(z)\right), \\
& \vdots \\
D^{m}(\lambda, \varphi) f(z) & =D(\lambda, \varphi)\left(D^{m-1}(\lambda, \varphi) f(z)\right),
\end{aligned}
$$

where $\lambda \geq \varphi \geq 0$ and $m \in \mathbb{N}_{0}=\mathbb{N} \cup\{0\}$.

If $f$ is given by (1.1), then from the definition of the operator $D^{m}(\lambda, \varphi) f(z)$ it is easy to see that

$$
D^{m}(\lambda, \varphi) f(z)=z+\sum_{n=2}^{\infty}[1+(\lambda \varphi n+\lambda-\varphi)(n-1)]^{m} a_{n} z^{n} .
$$

It should be remarked that the $D^{m}(\lambda, \varphi)$ is a generalization of many other linear operators considered earlier. In particular, for $f \in \mathcal{A}$ we have the following:

- $D^{m}(1,0) f(z) \equiv D^{m} f(z)$, the operator investigated by Sălăgean (see [23]).

- $D^{m}(\lambda, 0) f(z) \equiv D^{m}(\lambda) f(z)$, the operator studied by Al-Oboudi (see [2]).

- $D^{m}(\lambda, \varphi) f(z)$, the operator firstly considered for $0 \leq \varphi \leq \lambda \leq 1$, by Răducanu and Orhan (see [20]). The operator $D^{m}(\lambda, \varphi) f(z)$ is called Răducanu-Orhan operator. 
Definition 1.1. The generalized linear operator $L(m, \lambda, \varphi ; a, c): \mathcal{A} \rightarrow \mathcal{A}$ is given as

$$
\begin{aligned}
L(m, \lambda, \varphi ; a, c) f(z) & =\phi(a, c ; z) * D^{m}(\lambda, \varphi) f(z) \\
& =z+\sum_{n=2}^{\infty} \Phi_{n}^{m}(\lambda, \varphi) \frac{(a)_{n-1}}{(c)_{n-1}} a_{n} z^{n}
\end{aligned}
$$

where $\Phi_{n}^{m}(\lambda, \varphi)=[1+(\lambda \varphi n+\lambda-\varphi)(n-1)]^{m}, \lambda \geq \varphi \geq 0, m \in \mathbb{N}_{0}=\mathbb{N} \cup\{0\}$ and $a, c \neq-1,-2,-3, \ldots$

We note here some special cases:

(1) $L(0, \lambda, \varphi ; a, c) f(z)=L(a, c) f(z)$ is the Carlson-Shaffer linear operator [4].

(2) $L(0, \lambda, \varphi ; \delta+1,1) f(z), \delta \in \mathbb{N}_{0}$, is the Ruscheweyh derivative operator [22].

(3) $L(m, \lambda, \varphi ; 1,1) f(z), \lambda \geq \varphi \geq 0, m \in \mathbb{N}_{0}$, is extended Raducanu-Orhan operator [7].

(4) $L(m, \lambda, 0 ; 1,1) f(z), m \in \mathbb{N}_{0}$, is the Al-Oboudi linear operator [2].

(5) $L(m, 1,0 ; 1,1) f(z), m \in \mathbb{N}_{0}$, is the Sălăgean derivative operator [23].

Now, by making use of the extended linear differential operator $L(m, \lambda, \varphi ; a, c)$, we define a new subclass $Q(m, \lambda, \varphi, \beta ; a, c)$ of analytic functions.

Definition 1.2. Let $a, c$ be nonzero complex parameters such that $a, c \neq$ $-1,-2,-3, \ldots, \lambda \geq \varphi \geq 0, m \in \mathbb{N}_{0}=\mathbb{N} \cup\{0\}$. Also, suppose $0<\beta \leq 1$. A function $f$ given by (1.1) is said to be in the class $Q(m, \lambda, \varphi, \beta ; a, c)$ if

$$
\left|\arg \left(\frac{z(L(m, \lambda, \varphi ; a, c) f(z))^{\prime}}{L(m, \lambda, \varphi ; a, c) f(z)}\right)\right|<\frac{\pi}{2} \beta, z \in \mathcal{U} .
$$

This class includes a variety of well-known subclasses of $\mathcal{A}$. For example,

$$
\begin{aligned}
& Q(0, \lambda, \varphi, \beta ; a, a) \equiv S_{1}^{*}(\beta) \\
& =\left\{z \in A:\left|\arg \left(\frac{z f^{\prime}(z)}{f(z)}\right)\right|<\frac{\pi}{2} \beta, z \in \mathcal{U}\right\} ;[3] \\
& Q(0, \lambda, \varphi, \beta ; 2,1) \equiv K_{1}(\beta) \\
& =\left\{f \in A:\left|\arg \left(1+\frac{z f^{\prime \prime}(z)}{f^{\prime}(z)}\right)\right|<\frac{\pi}{2} \beta, z \in \mathcal{U}\right\} ;[3] \\
& Q(0, \lambda, \varphi, \beta, \delta+1,1) \equiv \tilde{R}_{\delta}(\beta) \\
& =\left\{f \in A:\left|\arg \left(\frac{z\left(D^{\delta} f(z)\right)^{\prime}}{D^{\delta} f(z)}\right)\right|<\frac{\pi}{2} \beta, z \in \mathcal{U}\right\}, \delta \geq 0 ;[6] .
\end{aligned}
$$

A function $f$ in $S_{1}^{*}(\beta)$ is called strongly starlike of order $\beta$. The class $K_{1}(\beta)$ consists of strongly convex functions of order $\beta$. These observations help us to conclude that the differential-integral representation given by (1.4) is a generalization of the Carlson-Shaffer operator in [4] and includes $S_{1}^{*}(\beta)$ and $K_{1}(\beta)$ studied by Brannan and Kirwan in [3]. 
In 1933, Fekete and Szegö [10] found the maximum value of $\left|a_{3}-\mu a_{2}^{2}\right|$ as a function of the real parameters $\mu$, for functions belonging to the class $S$. Since then, several researchers solved the Fekete-Szegö problem for various sublasses of the class of $S$ and related subclasses of functions in $\mathcal{A}$. See, for example [1], [5], [6], [7], [8], [11], [12], [13], [15], [16], [17], [18], [25]. In the present paper, we solve Fekete-Szegö problem for functional $\left|a_{3}-\mu a_{2}^{2}\right|$, where $\mu$ is real or complex when $f$ is in the family $Q(m, \lambda, \varphi, \beta ; a, c)$. In particular, our theorems contain corresponding results for various subclasses of strongly starlike and strongly convex and other several subclasses of $\mathcal{A}$.

2. Preliminary results. Let $P$ be the class of all analytic functions $P$ given by $p(z)=1+c_{1} z+c_{2} z^{2}+\ldots$ with $\operatorname{Re} p(z)>0$ for $z \in \mathcal{U}$. To prove our main results we need the following lemmas.

Lemma 2.1 ([19]). If $p(z)=1+c_{1} z+c_{2} z^{2}+\ldots$ is in $P$, then

(i) $\left|c_{n}\right| \leq 2$ for $n \geq 1$,

(ii) $\left|c_{2}-\frac{1}{2} c_{1}^{2}\right| \leq 2-\frac{\left|c_{1}\right|^{2}}{2}$.

Lemma 2.2. Let $a$ and $c$ be nonzero complex numbers with $a, c \neq-1$, $-2,-3, \ldots, \lambda \geq \varphi \geq 0$ and $m \in \mathbb{N}_{0}=\mathbb{N} \cup\{0\}$. If $f \in Q(m, \lambda, \varphi, \beta ; a, c)$ is given by (1.1) then

(i) $\left|a_{2}\right| \leq \frac{2 \beta|c|}{\Phi_{2}^{m}(\lambda, \varphi)|a|}$,

(ii) $\left|a_{3}\right| \leq \begin{cases}\frac{\beta|c||c+1|}{\Phi_{3}^{m}(\lambda, \varphi)|a||a+1|}, & \beta \leq \frac{1}{3}, \\ \frac{3 \beta^{2}|c||c+1|}{\Phi_{3}^{m}(\lambda, \varphi)|a||a+1|}, & \beta \geq \frac{1}{3} .\end{cases}$

Proof. Let $F(z):=L(m, \lambda, \varphi ; a, c) f(z):=z+A_{2} z^{2}+A_{3} z^{3}+\ldots$ Since

$$
\frac{z F^{\prime}(z)}{F(z)}=p^{\beta}(z), p \in P
$$

and so,

$$
\frac{z\left(1+2 A_{2} z+3 A_{3} z^{2}+\ldots\right)}{z+A_{2} z^{2}+A_{3} z^{3}+\ldots}=\left(1+c_{1} z+c_{2} z^{2}+\ldots\right)^{\beta},
$$

which implies that

$$
\begin{aligned}
z+2 A_{2} z^{2}+3 A_{3} z^{3}+\ldots & =z+\left(\beta c_{1}+A_{2}\right) z^{2} \\
+ & \left(\beta c_{2}+\frac{\beta(\beta-1) c_{1}^{2}}{2}+\beta c_{1} A_{2}+A_{3}\right) z^{3}+\ldots
\end{aligned}
$$

Equating the coefficients of $z^{2}$ and $z^{3}$, we have

$$
A_{2}=\beta c_{1} \text {, }
$$


since

$$
\begin{gathered}
A_{3}=\frac{\beta}{2}\left(c_{2}-\frac{c_{1}^{2}}{2}\right)+\frac{3}{4} \beta^{2} c_{1}^{2} . \\
F(z)=\phi(a, c ; z) * D^{m}(\lambda, \varphi) f(z)=z+\sum_{n=2}^{\infty} \Phi_{n}^{m}(\lambda, \varphi) \frac{(a)_{n-1}}{(c)_{n-1}} a_{n} z^{n} \\
=z+\sum_{n=2}^{\infty} \Phi_{n}^{m}(\lambda, \varphi) \frac{\Gamma(a+n-1) \Gamma(c)}{\Gamma(c+n-1) \Gamma(a)} a_{n} z^{n},
\end{gathered}
$$

so we have

$$
\beta c_{1}=\Phi_{2}^{m}(\lambda, \varphi) \frac{\Gamma(a+1) \Gamma(c)}{\Gamma(c+1) \Gamma(a)} a_{2} .
$$

This yields

$$
a_{2}=\frac{\beta c c_{1}}{a \Phi_{2}^{m}(\lambda, \varphi)} .
$$

In view of Lemma 2.1 (i) we have

$$
\left|a_{2}\right| \leq \frac{2 \beta|c|}{|a| \Phi_{2}^{m}(\lambda, \varphi)}
$$

On comparing the coefficients of $z^{3}$ in (2.3), we get

$$
A_{3}=\Phi_{3}^{m}(\lambda, \varphi) \frac{\Gamma(a+2) \Gamma(c)}{\Gamma(a) \Gamma(c+2)} a_{3}=\Phi_{3}^{m}(\lambda, \varphi) \frac{a(a+1)}{c(c+1)} a_{3} .
$$

Using (2.2), we obtain

$$
a_{3}=\frac{c(c+1)}{\Phi_{3}^{m}(\lambda, \varphi) a(a+1)}\left(\frac{\beta}{2}\left(c_{2}-\frac{c_{1}^{2}}{2}\right)+\frac{3}{4} \beta^{2} c_{1}^{2}\right) .
$$

Therefore, by applying Lemma 2.1 (ii), it follows that

$$
\left|a_{3}\right| \leq \frac{|c||(c+1)| \beta}{4 \Phi_{3}^{m}(\lambda, \varphi)|a||(a+1)|}\left\{4-\left|c_{1}\right|^{2}+3 \beta\left|c_{1}\right|^{2}\right\} .
$$

This inequality immediately proves the result.

3. Main results. We first consider the functional $\left|a_{3}-\mu a_{2}^{2}\right|$ for complex parameter $\mu$.

Theorem 3.1. Let $a$ and $c$ be complex parameters such that $a, c \neq 0,-1$, $-2,-3, \ldots, \lambda \geq \varphi \geq 0$ and $m \in \mathbb{N}_{0}=\mathbb{N} \cup\{0\}$. If $f \in Q(m, \lambda, \varphi, \beta ; a, c)$, $\beta \in(0,1]$ and $\mu$ is a complex parameter, then

$$
\left|a_{3}-\mu a_{2}^{2}\right| \leq \frac{\beta|c||c+1|}{\Phi_{3}^{m}(\lambda, \varphi)|a||a+1|} \max \left\{1, \frac{\beta v(\Phi, \mu ; a, c)}{\Phi_{2}^{2 m}(\lambda, \varphi)|a||c+1|}\right\},
$$

where $v(\Phi, \mu ; a, c)=3 \Phi_{2}^{2 m}(\lambda, \varphi) a(c+1)-4 \Phi_{3}^{m}(\lambda, \varphi) \mu c(a+1)$. 
Proof. From (2.4) and (2.5), it follows that

$$
\begin{aligned}
a_{3}-\mu a_{2}^{2} & =\frac{\beta c(c+1)}{2 \Phi_{3}^{m}(\lambda, \varphi) a(a+1)}\left(c_{2}-\frac{1}{2} c_{1}^{2}\right) \\
& +\frac{\beta^{2} c\left[3 \Phi_{2}^{2 m}(\lambda, \varphi) a(c+1)-4 \mu \Phi_{3}^{m}(\lambda, \varphi) c(a+1)\right]}{4 \Phi_{3}^{m}(\lambda, \varphi) \Phi_{2}^{2 m}(\lambda, \varphi) a^{2}(a+1)} c_{1}^{2} .
\end{aligned}
$$

Therefore,

$$
\begin{aligned}
\left|a_{3}-\mu a_{2}^{2}\right| & \leq \frac{\beta|c||c+1|}{2 \Phi_{3}^{m}(\lambda, \varphi)|a||a+1|}\left|c_{2}-\frac{1}{2} c_{1}^{2}\right| \\
& +\frac{\beta^{2}|c||v(\Phi, \mu ; a, c)|}{4 \Phi_{3}^{m}(\lambda, \varphi) \Phi_{2}^{2 m}(\lambda, \varphi)|a|^{2}|a+1|}\left|c_{1}\right|^{2} .
\end{aligned}
$$

In view of Lemma 2.1 (ii), we obtain

$$
\begin{aligned}
\left|a_{3}-\mu a_{2}^{2}\right| & \leq \frac{\beta|c||c+1|}{\Phi_{3}^{m}(\lambda, \varphi)|a||a+1|} \\
& +\frac{\beta|c|\left[\beta|v(\Phi, \mu ; a, c)|-\Phi_{2}^{2 m}(\lambda, \varphi)|a||c+1|\right]}{4 \Phi_{3}^{m}(\lambda, \varphi) \Phi_{2}^{2 m}(\lambda, \varphi)|a|^{2}|a+1|}\left|c_{1}\right|^{2} .
\end{aligned}
$$

Suppose $\beta|v(\Phi, \mu ; a, c)| \leq \Phi_{2}^{2 m}(\lambda, \varphi)|a||c+1|$. Then it immediately follows that

$$
\left|a_{3}-\mu a_{2}^{2}\right| \leq \frac{\beta|c||c+1|}{\Phi_{3}^{m}(\lambda, \varphi)|a||a+1|} .
$$

On the other hand, if $\beta|v(\Phi, \mu ; a, c)| \geq \Phi_{2}^{2 m}(\lambda, \varphi)|a||c+1|$, then using Lemma 2.1 (i), we have

$$
\begin{aligned}
& \left|a_{3}-\mu a_{2}^{2}\right| \leq \frac{\beta|c||c+1|}{\Phi_{3}^{m}(\lambda, \varphi)|a||a+1|} \\
& +\frac{\beta|c|\left[\beta|v(\Phi, \mu ; a, c)|-\Phi_{2}^{2 m}(\lambda, \varphi)|a||c+1|\right]}{\Phi_{3}^{m}(\lambda, \varphi) \Phi_{2}^{2 m}(\lambda, \varphi)|a|^{2}|a+1|} \\
& =\frac{\beta|a||c||c+1| \Phi_{2}^{2 m}(\lambda, \varphi)+\beta^{2}|c||v(\Phi, \mu ; a, c)|-\beta|a||c||c+1| \Phi_{2}^{2 m}(\lambda, \varphi)}{\Phi_{3}^{m}(\lambda, \varphi) \Phi_{2}^{2 m}(\lambda, \varphi)|a|^{2}|a+1|} \\
& =\frac{\beta^{2}|c||v(\Phi, \mu ; a, c)|}{\Phi_{3}^{m}(\lambda, \varphi) \Phi_{2}^{2 m}(\lambda, \varphi)|a|^{2}|a+1|} .
\end{aligned}
$$

The result immediately follows from (3.4) and (3.5).

Equality in (3.4) and (3.5) is attained, respectively, for functions in $Q(m, \lambda, \varphi, \beta ; a, c)$ given by

$$
\frac{z(L(m, \lambda, \varphi ; a, c) f(z))^{\prime}}{L(m, \lambda, \varphi ; a, c) f(z)}=\left(\frac{1+z^{2}}{1-z^{2}}\right)^{\beta}, \frac{z(L(m, \lambda, \varphi ; a, c) f(z))^{\prime}}{L(m, \lambda, \varphi ; a, c) f(z)}=\left(\frac{1+z}{1-z}\right)^{\beta} .
$$

In the next result we consider the cases where $\mu$ is a real parameter. 
Theorem 3.2. Let $a, c \in(0, \infty), \beta \in(0,1], \lambda \geq \varphi \geq 0$ and $m \in \mathbb{N}_{0}=$ $\mathbb{N} \cup\{0\}$. If $f \in Q(m, \lambda, \varphi, \beta ; a, c)$ and $f$ is given by (1.1) then for real $\mu$ we have

$$
\left|a_{3}-\mu a_{2}^{2}\right| \leq\left\{\begin{array}{c}
\frac{\beta^{2} c\left[3 a(c+1) \Phi_{2}^{2 m}(\lambda, \varphi)-4 \mu c(a+1) \Phi_{3}^{m}(\lambda, \varphi)\right]}{\Phi_{3}^{m}(\lambda, \phi) \Phi_{2}^{2 m}(\lambda, \phi) a^{2}(a+1)}, \\
\text { if } \mu \leq \frac{(3 \beta-1) a(c+1) \Phi_{2}^{2 m}(\lambda, \varphi)}{4 \beta c(a+1) \Phi_{3}^{m}(\lambda, \varphi)}, \\
\frac{\beta c(c+1)}{\Phi_{3}^{m}(\lambda, \varphi) a(a+1)}, \\
\text { if } \frac{(3 \beta-1) a(c+1) \Phi_{2}^{2 m}(\lambda, \varphi)}{4 \beta c(a+1) \Phi_{3}^{m}(\lambda, \varphi)} \leq \mu \leq \frac{(3 \beta+1) a(c+1) \Phi_{2}^{2 m}(\lambda, \varphi)}{4 \beta c(a+1) \Phi_{3}^{m}(\lambda, \varphi)}, \\
\frac{\beta^{2} c\left(4 \mu c(a+1) \Phi_{3}^{m}(\lambda, \varphi)-3 a(c+1) \Phi_{2}^{2 m}(\lambda, \varphi)\right)}{\Phi_{3}^{m}(\lambda, \varphi) \Phi_{2}^{2 m}(\lambda, \varphi) a^{2}(a+1)}, \\
\text { if } \mu \geq \frac{(3 \beta+1) a(c+1) \Phi_{2}^{2 m}(\lambda, \varphi)}{4 \beta c(a+1) \Phi_{3}^{m}(\lambda, \varphi)} .
\end{array}\right.
$$

Proof. In view of (3.3), we need to consider two main cases.

Case 1. Let $\mu \leq \frac{3 \Phi_{2}^{2 m}(\lambda, \varphi) a(c+1)}{4 \Phi_{3}^{m}(\lambda, \varphi) c(a+1)}$. Then (3.3) gives

$$
\begin{aligned}
& \left|a_{3}-\mu a_{2}^{2}\right| \leq \frac{\beta c(c+1)}{\Phi_{3}^{m}(\lambda, \varphi) a(a+1)} \\
& +\frac{\beta c\left[(3 \beta-1) a(c+1) \Phi_{2}^{2 m}(\lambda, \varphi)-4 \beta \mu c(a+1) \Phi_{3}^{m}(\lambda, \varphi)\right]}{4 \Phi_{3}^{m}(\lambda, \varphi) \Phi_{2}^{2 m}(\lambda, \varphi) a^{2}(a+1)}\left|c_{1}\right|^{2}
\end{aligned}
$$

and by using the fact that $\left|c_{1}\right| \leq 2$, we obtain

$$
\left|a_{3}-\mu a_{2}^{2}\right| \leq \frac{\beta^{2} c\left[3 a(c+1) \Phi_{2}^{2 m}(\lambda, \varphi)-4 \mu c(a+1) \Phi_{3}^{m}(\lambda, \varphi)\right]}{\Phi_{3}^{m}(\lambda, \varphi) \Phi_{2}^{2 m}(\lambda, \varphi) a^{2}(a+1)},
$$

provided that

$$
\mu \leq \frac{(3 \beta-1) a(c+1) \Phi_{2}^{2 m}(\lambda, \varphi)}{4 \beta c(a+1) \Phi_{3}^{m}(\lambda, \varphi)} .
$$

On the other hand, if

$$
\mu \geq \frac{(3 \beta-1) a(c+1) \Phi_{2}^{2 m}(\lambda, \varphi)}{4 \beta c(a+1) \Phi_{3}^{m}(\lambda, \varphi)},
$$

then the inequality (3.6) reduces to

$$
\begin{aligned}
\left|a_{3}-\mu a_{2}^{2}\right| & \leq \frac{\beta c(c+1)}{\Phi_{3}^{m}(\lambda, \varphi) a(a+1)} \\
& -\frac{\beta c\left[4 \mu \beta c(a+1) \Phi_{3}^{m}(\lambda, \varphi)-(3 \beta-1) a(c+1) \Phi_{2}^{2 m}(\lambda, \varphi)\right]}{4 \Phi_{3}^{m}(\lambda, \varphi) \Phi_{2}^{2 m}(\lambda, \varphi) a^{2}(a+1)}\left|c_{1}\right|^{2} \\
& \leq \frac{\beta c(c+1)}{\Phi_{3}^{m}(\lambda, \varphi) a(a+1)} .
\end{aligned}
$$

Case 2. Assume that $\mu \geq \frac{3 \Phi_{2}^{2 m}(\lambda, \varphi) a(c+1)}{4 \Phi_{3}^{m}(\lambda, \varphi) c(a+1)}$. In this case, note that

$$
v(\Phi, \mu ; a, c)=4 \Phi_{3}^{m}(\lambda, \varphi) \mu c(a+1)-3 \Phi_{2}^{2 m}(\lambda, \varphi) a(c+1)
$$


and (3.3) reduces to

$$
\begin{aligned}
& \left|a_{3}-\mu a_{2}^{2}\right| \leq \frac{\beta c(c+1)}{\Phi_{3}^{m}(\lambda, \varphi) a(a+1)} \\
& +\frac{\beta c\left[4 \beta \mu c(a+1) \Phi_{3}^{m}(\lambda, \varphi)-(3 \beta+1) a(c+1) \Phi_{2}^{2 m}(\lambda, \varphi)\right]}{4 \Phi_{3}^{m}(\lambda, \varphi) \Phi_{2}^{2 m}(\lambda, \varphi) a^{2}(a+1)}\left|c_{1}\right|^{2} .
\end{aligned}
$$

Again, using the fact that $\left|c_{1}\right| \leq 2$, we obtain

$$
\left|a_{3}-\mu a_{2}^{2}\right| \leq \frac{\beta^{2} c\left[4 \mu c(a+1) \Phi_{3}^{m}(\lambda, \varphi)-3 a(c+1) \Phi_{2}^{2 m}(\lambda, \varphi)\right]}{\Phi_{3}^{m}(\lambda, \varphi) \Phi_{2}^{2 m}(\lambda, \varphi) a^{2}(a+1)},
$$

where we have also used the condition that

$$
\mu \geq \frac{(3 \beta+1) a(c+1) \Phi_{2}^{2 m}(\lambda, \varphi)}{4 \beta c(a+1) \Phi_{3}^{m}(\lambda, \varphi)} .
$$

On the other hand, if

$$
\mu \leq \frac{(3 \beta+1) a(c+1) \Phi_{2}^{2 m}(\lambda, \varphi)}{4 \beta c(a+1) \Phi_{3}^{m}(\lambda, \varphi)},
$$

then (3.7) yields

$$
\begin{aligned}
\left|a_{3}-\mu a_{2}^{2}\right| & \leq \frac{\beta c(c+1)}{\Phi_{3}^{m}(\lambda, \varphi) a(a+1)} \\
& -\frac{\beta c\left[(3 \beta+1) a(c+1) \Phi_{2}^{2 m}(\lambda, \varphi)-4 \mu \beta c(a+1) \Phi_{3}^{m}(\lambda, \varphi)\right]}{4 \Phi_{3}^{m}(\lambda, \varphi) \Phi_{2}^{2 m}(\lambda, \varphi) a^{2}(a+1)}\left|c_{1}\right|^{2} \\
& \leq \frac{\beta c(c+1)}{\Phi_{3}^{m}(\lambda, \varphi) a(a+1)} .
\end{aligned}
$$

Finally, we observe that

$$
\begin{aligned}
\frac{(3 \beta-1) a(c+1) \Phi_{2}^{2 m}(\lambda, \varphi)}{4 \beta c(a+1) \Phi_{3}^{m}(\lambda, \varphi)} \leq \mu & \leq \frac{3 a(c+1) \Phi_{2}^{2 m}(\lambda, \varphi)}{4 c(a+1) \Phi_{3}^{m}(\lambda, \varphi)} \\
& \leq \frac{(3 \beta+1) a(c+1) \Phi_{2}^{2 m}(\lambda, \varphi)}{4 \beta c(a+1) \Phi_{3}^{m}(\lambda, \varphi)}
\end{aligned}
$$

Thus the proof is complete.

Corollary 3.3. Let $a, c \in(0, \infty), \lambda \geq \varphi \geq 0, m \in \mathbb{N}_{0}=\mathbb{N} \cup\{0\}$ and

$$
0<\beta \leq \frac{3 a(c+1) \Phi_{2}^{2 m}(\lambda, \varphi)}{9 a(c+1) \Phi_{2}^{2 m}(\lambda, \varphi)-8 c(a+1) \Phi_{3}^{m}(\lambda, \varphi)} .
$$

If $f \in Q(m, \lambda, \varphi, \beta ; a, c)$ and $f$ is given by (1.1), then

$$
\left|a_{3}\right|-\left|a_{2}\right| \leq \frac{\beta c(c+1)}{\Phi_{3}^{m}(\lambda, \varphi) a(a+1)} .
$$




\section{Proof. Since}

$$
\frac{(3 \beta-1) a(c+1) \Phi_{2}^{2 m}(\lambda, \varphi)}{4 c(a+1) \beta \Phi_{3}^{m}(\lambda, \varphi)} \leq \frac{2}{3}
$$

for

$$
\beta \leq \frac{3 a(c+1) \Phi_{2}^{2 m}(\lambda, \varphi)}{9 a(c+1) \Phi_{2}^{2 m}(\lambda, \varphi)-8 c(a+1) \Phi_{3}^{m}(\lambda, \varphi)}
$$

and

$$
\left|a_{3}\right|-\left|a_{2}\right| \leq\left|a_{3}-\frac{2}{3} a_{2}^{2}\right|+\frac{2}{3}\left|a_{2}\right|^{2}-\left|a_{2}\right|,
$$

from Theorem 3.2 it follows that

$$
\left|a_{3}\right|-\left|a_{2}\right| \leq \frac{\beta c(c+1)}{\Phi_{3}^{m}(\lambda, \varphi) a(a+1)}+\frac{2}{3}\left|a_{2}\right|^{2}-\left|a_{2}\right| .
$$

Setting $\left|a_{2}\right|:=x \in[0,2 \beta c / a]$, we can write

$$
\left|a_{3}\right|-\left|a_{2}\right| \leq \frac{\beta c(c+1)}{\Phi_{3}^{m}(\lambda, \varphi) a(a+1)}+\frac{2}{3} x^{2}-x:=\Omega(x) .
$$

Since $\Omega(x)$ attains its maximum value at $x=0$, the result follows.

Acknowledgement 1. The work presented here was done when the first author visited Atatürk University and the second author visited Ohio State University. The first author's visit was supported by Kent State University.

Acknowledgement 2. Authors would like to thank the referee for thoughtful comments and suggestions.

\section{REFERENCES}

[1] Abdel-Gawad, H. R., Thomas, D. K., Fekete-Szegö problem for strongly close-toconvex function, Proc. Amer. Math. Soc. 114 (2) (1992), 345-349.

[2] Al-Oboudi, F. M., On univalent functions defined by a generalized Sălăgean operator, Int. J. Math. Math. Sci., no. 25-28 (2004), 1429-1436.

[3] Brannan D. A., Kirwan, W. E., On some classes of bounded univalent functions, J. London Math. Soc. 2 (1) (1969), 431-443.

[4] Carlson, B. C., Shaffer, D. B., Starlike and prestarlike hypergeometric functions, SIAM J. Math. Anal. 15 (1984), 737-745.

[5] Çağlar, M., Deniz, E., Orhan, H., Coefficient bounds for a subclass of starlike functions of complex order, Appl. Math. Comput. 218 (2011), 693-698.

[6] Darus, M., Akbarally, A., Coefficient estimates for Ruscheweyh derivatives, Int. J. Math. Math. Sci. 36 (2004), 1937-1942.

[7] Deniz, E., Orhan, H., The Fekete-Szegö problem for a generalized subclass of analytic functions, Kyungpook Math. J. 50 (2010), 37-47.

[8] Deniz, E., Çağlar, M., Orhan, H., The Fekete-Szegö problem for a class of analytic functions defined by Dziok-Srivastava operator, Kodai Math. J. 35 (2012), 439-462.

[9] Dziok, J., Classes of functions defined by certain differential-integral operators, J. Comput. Appl. Math. 105 (1999), 245-255.

[10] Fekete, M., Szegö, G., Eine Bermerkung uber ungerade schlichte funktionen, J. London Math. Soc. 8 (1933), 85-89. 
[11] Frasin, B., Darus, M., On Fekete-Szegö problem using Hadamard product, Int. J. Math. Math. Sci. 12 (2003), 1289-1295.

[12] Goel, R. M., Mehrok, B. S., A coefficient inequality for certain classes of analytic functions, Tamkang J. Math. 22 (2) (1995), 153-163.

[13] Koeghe, F. R., Merkes, E. P., A coefficient inequality for certain classes of analytic functions, Proc. Amer. Math. Soc. 20 (1969), 8-12.

[14] Lashin, A. Y., Starlike and convex functions of complex order involving a certain linear operator, Indian J. Pure Appl. Math. 34 (7) (2003), 1101-1108.

[15] Orhan, H., Kamali, M., On the Fekete-Szegö problem, Appl. Math. Comput. 144 (2003), 181-186.

[16] Orhan, H., Raducanu, D., Fekete-Szegö problem for strongly starlike functions associated with generalized hypergeometric functions, Math. Comput. Modelling 50 (2009), 430-438.

[17] Orhan, H., Yağmur, N., Deniz, E., Coefficient inequality for a generalized subclass of analytic functions, Bull. Transilv. Univ. Braşov Ser. III 4(53), no. 1 (2011), 51-57.

[18] Orhan, H., Deniz, E., Çağlar, M., Fekete-Szegö problem for certain subclasses of analytic functions, Demonstratio Math. 45, no. 4 (2012), 835-846.

[19] Pommerenke, Ch., Univalent Functions, Vandenhoeck and Ruprecht, Gottingen, 1975.

[20] Răducanu, D., Orhan, H., Subclasses of analytic functions defined by a generalized differential operator, Int. J. Math. Anal. (Ruse) 4 (1) (2010), 1-15.

[21] Ravichandran, V., Kumar, S. S., On a class of analytic functions involving CarlsonShaffer linear operator, Riv. Math. Univ. Parma 7 (3) (2004), 35-48.

[22] Ruscheweyh, S., New criteria for univalent functions, Proc. Amer. Math. Soc. 49 (1975), 109-115.

[23] Sălăgean, G. S., Subclasses of univalent functions, Complex analysis - fifth Romanian-Finnish seminar, Part 1 (Bucharest, 1981), Lecture Notes in Math. 1013, Springer, Berlin, 1983, 362-372.

[24] Srivastava, H. M., Owa, S. (Eds.), Current Topics in Analytic Fuction Theory, World Scientific Publishing, New Jersey, 1992.

[25] Srivastava, H. M., Mishra, A. K., Das, M. K., The Fekete-Szegö problem for a subclass of close-to-convex functions, Complex Variable Theory Appl. 44 (2) (2001), 145-163.

Om P. Ahuja

Department of Mathematical Sciences

Kent State University

Burton

Ohio 44021-9500

U.S.A.

e-mail: oahuja@kent.edu
Halit Orhan

Department of Mathematics

Faculty of Science

Ataturk University

Erzurum, 25240

Turkey

e-mail: orhanhalit607@gmail.com

Received May 24, 2012 